Journal of Thermal Engineering, Vol. 5, No. 2, Special Issue 9, pp. 22-28, February, 2019

Yildiz Technical University Press, Istanbul, Turkey

\title{
A REVIEW OF GENERAL AND MODERN METHODS OF AIR PURIFICATION
}

\author{
Aditya Roy ${ }^{1,}$, Chetan Mishra ${ }^{1}$, Sarthak Jain ${ }^{1}$, Naveen Solanki ${ }^{1}$
}

\begin{abstract}
The air purifier industry has seen a growth in terms of demand and sales lately. All credit goes to massive industrialization in developing countries such as India and China. As a result, a lot of research has been focused into the various methods of purifying air. The most harmful of the pollutants are PM 2.5 particulates and NOx emissions. The aim has been to bring down the costs without compromising on efficiency as efficient air purification is an expensive deal. This article presents a study of the current scenario of the problems of air pollution. Severity of the issues have been highlighted. A compilation of the most common and significant methods of purifying air such as those employing the use of HEPA filters, electrostatic smoke precipitators, activated carbon and UV light has been presented and their use in air purifiers manufactured by OEMs has been mentioned. Some of the most modern methods of purifying air such as those using transparent PAN filters, photochemical materials, soy proteins and silk nanofibrils have been studied and reviewed. It has been found that these methods provide an attractive and economical pathway of filtering out PM 2.5 when compared to the conventional HEPA filters.
\end{abstract}

\section{Keywords: Air Purifier, PM 2.5, NOx Emissions, Bio Materials}

\section{INTRODUCTION}

Air pollution has become one of the most significant and deadliest causes of deaths today. The World Health Organization (WHO) stated that around 6.5 million deaths were caused by air pollution in the year 2012 translating to $11.6 \%$ of total global deaths [1]. WHO states that air pollution is the cause of one-third of deaths caused due to stroke, lung cancer, and chronic respiratory diseases. Approximately, $92 \%$ of the world's population is exposed to air pollution levels that are beyond the permissible limits prescribed by WHO [2]. There are three basic strategies to tackle indoor air pollution [3]. The first is Source Control wherein, the source of pollutants is considered, and steps are taken to eliminate or decrease pollutants right from the source. If feasible enough and practically possible, this is the most effective method of reducing air pollutants. Ventilation is another method of reducing indoor air pollution. The fundamental for attaining a good ventilation system is very simple. An efficient system of exchange of air between inside and outside of a closed space needs to be ensured. Infiltration refers to the induction of outside air into a closed space through openings, joints or cracks in walls. Infiltration falls under natural ventilation which on a broader spectrum refers to the inclusion of outside air into a room or building without the aid of any external promoter. The third method of reducing indoor air pollution is usage of air purification systems also known as air purifiers. Air purifiers have been in trend lately because of their high efficiency of purifying polluted air. There are various OEMs that manufacture air purifiers with different technologies and mechanisms of air purification. It can be stated here that an air purifier is not self-sufficient to tackle indoor air pollution but requires a good ventilation system in conjunction. The job of an air purifier is twofold reduction of pollutants, they being particulate matter (PM) and gaseous pollutants. The most common air purification media are being discussed here.

Household air pollution is one of the areas that need immediate attention. There are different sources of indoor air pollution like tobacco smoking, fuel used for cooking, use of pesticides and chemical which causes disabilities among people. Household air pollution affects mostly the respiratory system and further leads to cancer. Apte and Salvi [4] helped in identifying different household air pollutants and their health implications and strategies to curb household air pollution. Indoor air quality (IAQ) is one of the major factors for the building owners and facility manager. IAQ has to be assessed properly before building any structure. Advanced techniques like moisture control, This paper was recommended for publication in revised form by Regional Editor Sandip A. Kale ${ }^{1}$ Department of Mechanical and Automation Engineering, Maharaja Agrasen Institute of Technology, Delhi, India ${ }^{\star}$ E-mail address: aditya.roy1510@gmail.com

Orcid id: 0000-0003-1913-9355, 0000-0002-1003-2132, 0000-0001-5950-1625, 0000-0001-8011-0776

Manuscript Received 13 January 2018, Accepted 31 March 2018 
pressurization strategies were developed to better manage IAQ. Shrimandilkar [5] provided an analysis of relationships between IAQ technologies and construction practices. Recommendations are made for monitoring moisture in buildings during construction. There is a serious concern over the pollution emitted from burning of various fuels like cow dung, wood etc. for cooking purposes. IAQ was tested to estimate the emissions of particulate matter and Volatile Organic Compounds (VOCs). The results revealed that cow dung is most polluting fuel with maximum emissions of PM and VOCs. The solution to this is LPG fuels which is a healthier option as compared to the conventional fuels. Gautam et al. [6] claimed that the level of pollution can also be decreased by providing proper ventilation systems, replacing traditional stoves with improved efficient stoves.

Bolashikov and Melikov [7] illustrated different methods and technologies for indoor air purification and disinfection from microorganisms and viruses for HVAC systems and their applications. Different air purification methods like Dilution, Filtration, Photocatalytic oxidation etc. were studied and their characteristics were compared with the air purification techniques used today. Some oils can be used as antimicrobial and anti-fungal agents for reducing the number of pathogens and thus helping in disinfection of air. Carbon dioxide is one of the vital resources one needs but on the other hand if its indoor volume increases, it can pose some threats. Hu et al. [8] illustrated the use of sorption type air filters along with coconut based granular activated carbon to prevent Carbon Dioxide. The adsorption of Carbon Dioxide can be increased by using Magnesium Oxide and Calcium Oxide. This paper also explains the surface area analysis and calculation of initial efficiency, breakthrough curves and pressure drop to validate the results.

\section{GENERAL METHODS OF AIR PURIFICATION}

Vijayan et al. [9] studied the positive impact of mechanical air filters on indoor air pollution. Mechanical air filters refer to a meshed structure through which contaminated air is made to pass. The pore size of mesh is the most important factor for deciding the efficiency of mechanical filters. Contaminants having sizes greater than the mesh pore size are unable to pass and thus get separated leading to air purification. Such air filters are responsible for filtering out PM pollutants from indoor air. High Efficiency Particulate Air (HEPA) filters are the most used filtration media in today's air purifiers for filtering out PM. They have an efficiency of removing $99.97 \%$ of particulate matter of size less than 0.3 microns from contaminated air ensuring a flow rate anywhere between 150-400 cubic feet per minute depending on the clogging of their pores. HEPA filters appear in the form of pleated paper which is actually a very dense network of glass fibers. HEPA filters work on the principle of Brownian motion pertaining to which, there are three different mechanisms of trapping dust particles viz., impact, interception and diffusion. It is customary to use a pre-filter with HEPA filters in order to increase their life between subsequent replacements. Pre-filters are essentially a fiber mesh of comparatively larger pore sizes. Pre-filters are essential to trap the relatively larger particulates present in air so that they do not unnecessarily clog the HEPA pores.

Electronic filters are not filters essentially. Instead, they create an electric field for ionizing the particles passing through it. Electrostatic Smoke Precipitators (ESP) are electronic air filters which work on the principle of corona discharge. It is to be noted that the working of ESP requires a supply of high input voltage. There are two oppositely charged terminals which may be in the form of plates, wires or bars. Contaminated air is made to pass through the negatively charged terminal. As a result, the pollutants present in air get negatively charged. The flow of air is made in such a way that it passes through the positively charged terminal after moving past its negatively charged counterpart. All the negatively charged contaminants get attracted to the positively charged plates. In this way, air purification is achieved. An important advantage of ESP is that any size of particulate matter may be removed from polluted air unlike mechanical (HEPA) filters that pose a restriction on the pore size of filtrable particles. All that matters in an ESP is the supply voltage. The higher the supply voltage, finer the size of filtrable contaminants. ESP is thus capable of filtering out PM 2.5 contaminants. Zukeran et al. [10] studied the role of ESP in filtering out smoke being emitted by incense sticks. The dependence of efficiency on supply voltage was plotted by varying the same from 9 to 30 Kilo Volts. 


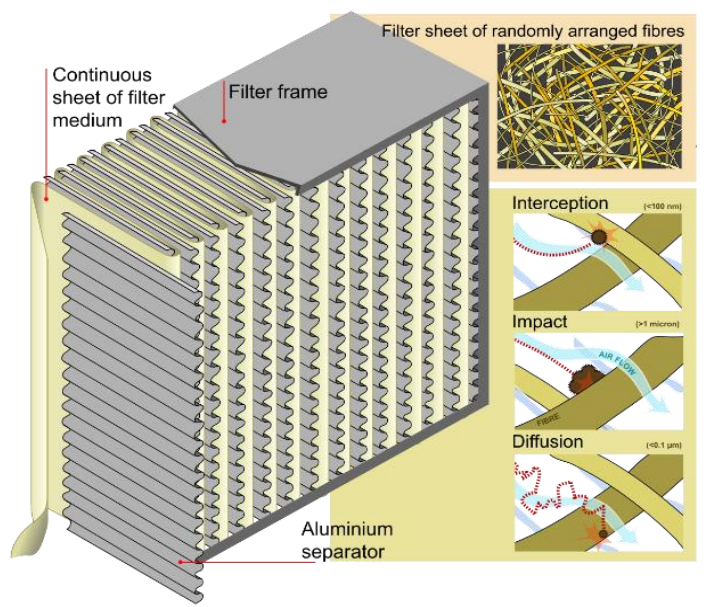

Figure 1. HEPA air filter and its particle trapping mechanisms [13]

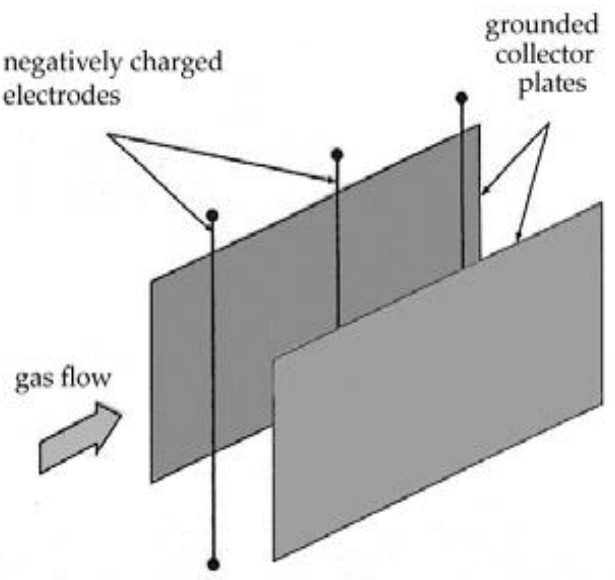

Figure 2. Schematic diagram of electrostatic smoke precipitator [21]

Chambre [11] studied the effects of an activated carbon layer in the path of impure air. Activated carbon is used for filtering out the gaseous pollutants present in air. There may be two forms of activated carbon viz., granular and bonded as studied by Chambre. It exhibits a high degree of porosity and a large surface area for interaction with pollutants. This enables it to attain very good adsorption characteristics. the principal governing the operation of activated carbon is based upon the concept of adsorption. There are two main types of adsorption that take place viz., physical adsorption and chemisorption. The preliminary process is physical adsorption wherein, Brownian motion of molecules is the dominating factor. Van der Waals' forces promote adsorption in which case, the gas molecules interact with the pores and get trapped as shown in Figure 3. Chemisorption follows the process of physical adsorption. The remaining gas pollutants react with chemical media of the activated carbon layer to form a compound which get trapped in the filter material. Results showed that granular activated carbon filter performed better than bonded activated carbon in terms of retention of mass of isopropanol at a given run time before reaching $1 \%$ of threshold limit value.

The process of air purification using Ultra Violet (UV) light as a filtration medium is very simplistic in nature. A source of UV radiations is selected appropriately which emits UV radiations in the surrounding medium. Polluted air is made to pass through this medium as a result of which, the bacteria and pathogens present in air get filtered out. UV filters are responsible for removing only microbes and pathogens from air and do not contribute in PM filtration. Kujundzic et al. [12] studied the mechanism and effects of air purification using UV light in great detail. A setup combining HEPA filter and UV light filter mounted on the ceiling was used for analysis. The purpose of the study was to analyze the effectiveness of UV air filters when used in conjunction with HEPA filters. The setup reduced the total bacteria in the target space by $12 \%$ and $76 \%$ over a span of two years respectively.

\section{REVIEW OF MODERN METHODS OF AIR POLLUTION}

This section presents a consolidated review of some of the most modern advancements in the field of air purification.

\section{Transparent PAN filter}

Nearly all air purifiers require some kind of power supply for their operation. Liu et al. [14] researched upon an air filter that is transparent in nature and composed of Nano-polymer materials to filter out PM 2.5 pollutants which are most harmful for the human body. Polyacrylonitrile (PAN) filters are essentially nanofibers that work on the principle of particle adhesion for curbing PM 2.5 pollutants. It was realized that windows in a house are the best sources for ventilation and it is always advisable to live in a ventilated environment in situations of air pollution.

Pertaining to this, it may be said that windows remain an unutilized resource as technically, there is no means of air purification here. PAN filters take advantage of this loophole by introducing a layer of transparent filter material 
which makes particulate matter adhere to its surface at the same time allowing natural light to enter the room with additional benefits of good optical transparency, low resistance to air flow, light weight and high filtering efficiency. Experimental results show an efficiency of more than 95\% for PM 2.5 filtration which varies according to the surface chemistry of production of the polymer filter as well as the transparency to be achieved. Electrospinning process is employed in the production of PAN filter wherein, the polymer solution is accumulated in a syringe as shown in Figure 4. A certain voltage is supplied at the tip of the syringe due to which an electrical force is generated. This force pulls the polymer solution in nanofiber form to be deposited on a grounded collector which in this experiment was a metallic mesh. The required network of air filter layer is formed owing to the deposition of fiber on the mesh as a result of the electrical force distribution. The chosen materials for experimentation were polyacrylonitrile (PAN), polyvinyl pyrrolidine (PVP), polystyrene (PS), polyvinyl alcohol (PVA) and polypropylene (PP) but PAN displayed the best results. The transparent filtration technology proves to be an attractive alternative to the most commonly used methods of PM 2.5 filtration such as HEPA filtration. These filters also have potential in bringing down the production and running costs significantly when compared to technologies like HEPA and electrostatic smoke precipitators.

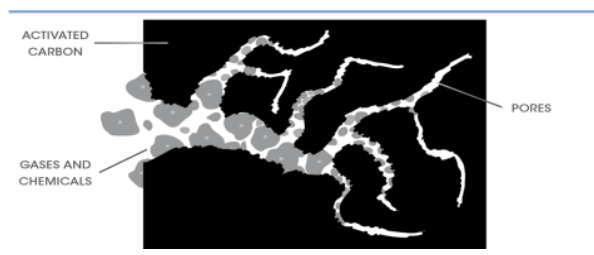

Figure 3. Adsorption mechanism of activated carbon [11]

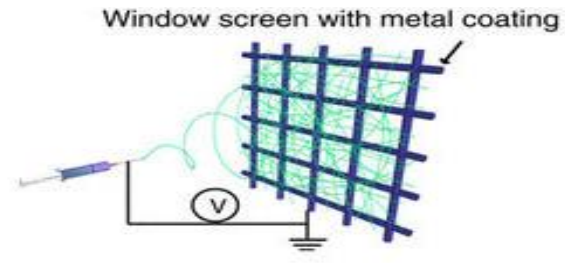

Figure 4. Process of electrospinning for making PAN filter [14]

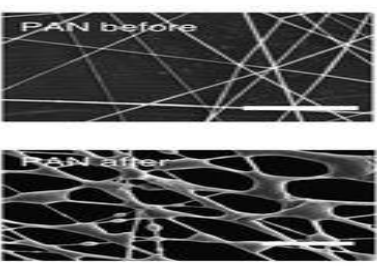

Figure 5. PAN filter before and after PM 2.5 filtration [14]

\section{Photocatalytic materials and technologies}

Major types of photocatalysts used in air purification system are complex metal oxides, binary metal oxides, metal sulphides and metal free materials. Photocatalytic materials and technology for air purification are based on the principle that radiation of suitable wavelength can be absorbed by many semiconductors that can facilitate the reaction of the reactive oxygen species which can decompose air pollutants. The electronic band structure of photocatalytic material consists of the valance band and conduction band which are separated by an energy band gap. According to Ren et al. [15], when light falls on the semiconductor, it absorbs the energy of photons of sufficient energy, the electron in the valence band can jump to conduction band hence leaving holes in valence band. Both of these charges are now available to diffuse to the surface of this photocatalyst here they can react. The presence of surface active sites facilitates the adsorption of environmental species then the redox reaction can take place as electron can react with electron acceptor and holes can react with electrons donors. When these radicals detach from the surface and become mobile, they can form reactive oxygen species, which are reactive owing to their unsaturated bonding. The reactive oxygen species can migrate away from photocatalytic surface. Reactive oxygen species which typically are $\mathrm{OH}^{-}, \mathrm{O}_{2}^{-}$ can decompose organic and inorganic pollutants into Carbon Dioxide, water and other inorganic products such as Nitrogen. All photocatalytic materials can be excited by UV radiations, those which are excited by visible light show absorption over limited range of visible light wavelength. Almost all commercial air purification systems use UV light sources with wavelengths in the range of 351 nanometers to 400 nanometers.

A laboratory photoreactor with a lamp producing 365 nanometer wavelength and a Titanium Dioxide coated fiberglass mesh was used and a removal rate of 5-10 micro mole per watt hour of Carbon Monoxide, N-Octane and Pyridine was achieved and consumed by the lamp with concentration of 50-2000 parts per million concentration with a flow rate of 25-50 liters per hour. The reduction of Benzene, Toluene \& Xylene was also observed by a factor of 2 to 3 in an ordinary room. The adding of Ozone in Oxygen increases in mineralization percentage of N-Octane. Pichat et al. [16] claimed that this property of Ozone can increase the application and presence of photocatalytic air purification in the industry. 
Heterogeneous photocatalysis is one of the main methods of air purification. The most commonly used heterogeneous photocatalyst is Titanium Dioxide (TiO2) and it can be mixed with building materials like cement to increase its purification capacity. It helps in removal of NOx emissions. Anne Beeldens [17] explained the chemistry behind air purification and graphs were plotted between time and concentration of NOx emissions to validate the results.

The emissions from vehicles leads to poor air quality. Several photocatalysts like TiO2 were used in asphalt pavement for purifying ambient air from vehicle's emissions. Artificial neural network (ANN) and neuro-fizzy (NF) models were built to predict NOx concentration in air as a function of traffic count and climatic conditions before and after application of $\mathrm{TiO} 2$ on pavement surface. Asadi et al. [18] selected these models because they were capable of modeling non-linear relationships. Results indicated that traffic level, relative humidity and solar radiation had the most influence on efficiency and NF model provided more accurate results than ANN.

\section{Composite membranes using soy proteins}

Conventional air filters are not efficient to address the increasing PM pollution problems in developing countries. When they are disposed, they can cause secondary pollution. Liu et al. [19] zeroed upon the fact that biomaterials like soybean can provide a cost effective and biodegradable method to this problem. Protein derived from soybean is available in three forms namely soy protein isolate, soy protein concentrates and defatted soy flour. Soy protein isolate (SPI) is the best of the lot due to its high protein content (90\%) and its low cost. SPI can attract PM particles due to electrostatic force of attraction and dipole attractions. SPI combines with Bacterial cellulose (BC), which is synthesized during fermentation of Gluconacetobacter Xylinus and has a 3D nanonetwork which helps in filtering PM particles physically (Figure 6). Due to large intermolecular interactions in SPI, they aggregate and form particles of 20-50 microns. Denaturation process is done in the presence of acrylic acid, to destroy the intermolecular interactions between SPI molecules and to reduce the particle size of SPI. This enables SPI to interact with new PM particles in air. By using SPI and BC in an appropriate ratio, efficiencies in the range of $99.4 \%$ and $99.95 \%$ can be achieved for PM 2.5 and PM 10 respectively, while maintaining a very high air penetration rate of $92.63 \%$.

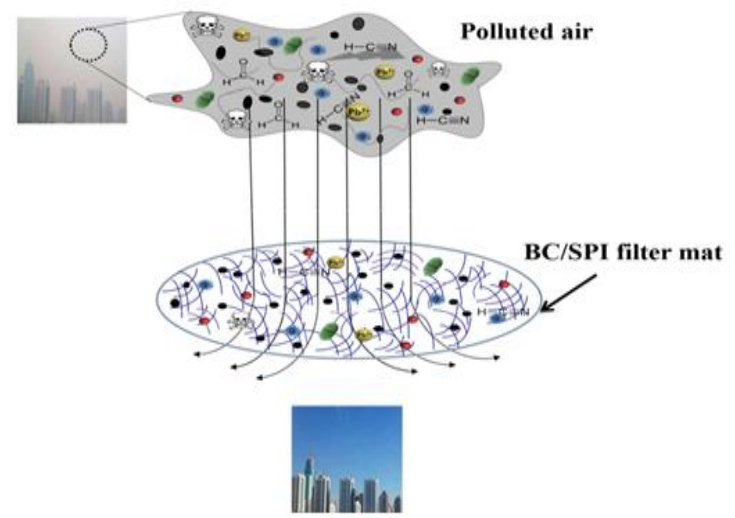

Figure 6. Capturing of various types of pollutants by bio-based materials via active fiber surfaces [19]

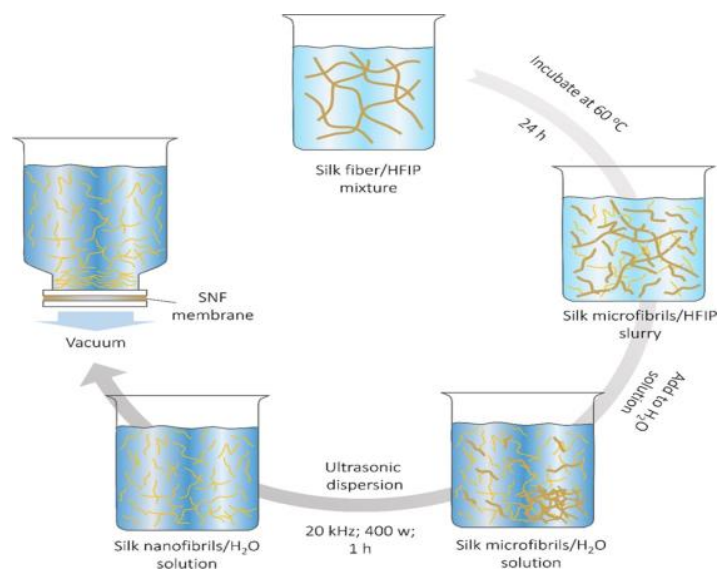

Figure 7. Process used to design ultrathin SNF membranes [20]

\section{Ultrathin silk nanofibril membranes}

Ling et al. [20] found that the main problem associated with non-porous membranes are their low permeation flux. To deal with this problem, we need a cost effective ultrathin filtration membrane with a good permeable flux. A possible solution to this is, Silk nanofibrils (SNFs), which is exfoliated from native Bombyx mori (B.mori) silk fibers to retain structure and physical properties. Firstly, the degummed B.mori were immersed in Hexafluoro Isopropanol (HFIP) solution, incubated at $60^{\circ} \mathrm{C}$ degrees on Celsius scale and after 24 hours, resultant silk microfibrils (SMF) pulp blend was dried. After that undissolved silk was removed by continuous shaking and then SMF was sonicated at 120 
micrometer amplitude and $20 \mathrm{kHz}$ frequency at intervals of 10s. Finally, SNFs were assembled to ultrathin membranes, using a vacuum filtration process. Fourier transform infrared spectroscopy (FTIR) was used to access structural details of the SNF membrane. Figure 7 shows the process. Pore size of the membrane can be varied by choosing appropriate filtration volume and concentration of SNF dispersion. The separation performance of SNF membranes was measured through pressure-driven filtration.

\section{CONCLUSION}

Air pollution has become one of the largest sources of all reported deaths in the past few years and it would not be wrong to say that mankind has been shaken by its very existence. Air pollutants can be subdivided into categories but the most harmful of them all are particulate matters of sizes less than 2.5 microns and NOx emissions. Human body is equipped suitably to trap the particulate matter entering while we exhale but the problem occurs in dealing with PM 2.5 as their size becomes so minute that it is not technically possible for our body to trap them. In an effort to find a solution to this problem, HEPA filters were designed which are a multi layered structure of glass fibers in order to trap the PM 2.5 particles. They do promise a very high efficiency of $99.97 \%$ but all of this comes at a cost owing to the expensive and intensive process of production. A very attractive method of curbing industrial air pollution is to use electrostatic smoke precipitators, their advantage lying in the fact that they can nearly filter out every possible category of air pollutants starting from particulates to odor. The downside is that they require a very high voltage and current supply making their use unfeasible for indoor home applications. UV light proves to be a suitable method to fight the bacterial pollutants that may be present provided the wavelength of light being used is not harmful for humans. Activated carbon is one of the conventional methods of purification with little to no disadvantages. This paper presents a compilation of some of the most recent methods of purifying air which are cost effective and efficient at the same time. Transparent PAN filters bank on the fact that windows provide a good amount of surface area for introducing a layer of filter without sacrificing natural light and ventilation. Photocatalytic materials provide a novel pathway to curb NOx emissions. Soy proteins and silk nanofibrils provide a method of utilizing natural resources to purify air. Future scope of work lies in further research that needs to be carried out on how these methods can be combined for the various categories of pollutants to be used in an air purifier for bringing out one of the most economical solutions to fight this vicious problem in hand.

\section{NOMECLATURE}

PM Particulate Matter

UV Ultra Violet

HEPA High Efficiency Particulate Air

OEM Original Equipment Manufacturer

PAN Poly Acrylo Nitrile

WHO World Health Organization

IAQ Indoor Air Quality

VOC Volatile Organic Compound

LPG Liquified Petroleum Gas

HVAC Heat Ventilation and Air Conditioning

ESP Electrostatic Smoke Precipitator

PVP Poly Vinyl Pyrrolidine

PS Poly Styrene

PVA Poly Vinyl Alcohol

PP Poly Propylene

$\mathrm{TiO}_{2} \quad$ Titanium Dioxide

ANN Artificial Neural Network

NF Neuro-Fizzy

SPI Soy Protein Isolate

BC Bacterial Cellulose

B mori Bombyx mori

HFIP Hexafluoro Isopropanol

SMF Silk Micro Fibrils 
SNF Silk Nano Fibrils

FTIR Fourier Transform Infrared Spectroscopy

\section{REFERENCES}

[1] http://www.who.int/mediacentre/news/releases/2016/air-pollution-estimates/en/

[2] http://apps.who.int/iris/bitstream/10665/250141/1/9789241511353-eng.pdf

[3] Indoor Air Quality: A summary of available information Residential Air Cleaners 2009 EPA 402-F-09-002

[4] Apte, K. and Salvi, S. (2016). Household air pollution and its effects on health. F1000 Research: F1000 Faculty Rev - 2593, 5.

[5] Shrimandilkar, P.P. (2013). Indoor air quality monitoring for human health. International Journal of Modern Engineering Research, 3(2), 891-897.

[6] Gautam, S.K., R., Suresh, Sharma, V.P., \& Sehgal, M. (2013). Indoor air quality in the rural India. Management of Environmental Quality: An International Journal, 24(2), 244-255.

[7] Bolashikov, Z. D., \& Melikov, A. K. (2007). Methods for indoor air disinfection and purification from airborne pathogens for application in HVAC systems. In Proceedings of The sixth international conference on indoor air quality, ventilation \& energy conservation in buildings (pp. 565-573).

[8] Hu, S., Shiue, A., Chang, S., Chang, Y., Tseng, C., Mao C., Hsieh, A., \& Chan, A. (2017). Removal of carbon dioxide in the indoor environment with sorption-type air filters. International Journal of Low-Carbon Technologies, 12(3), 330-334.

[9] Vijayan, V.K., Paramesh H., Salvi, S.S., \& Dalal, A.A.K. (2015). Enhancing indoor air quality, "The air filter advantage Lung India. Official Organ of Indian Chest Society, 32(5), 473-479.

[10] Zukeran, A., Chang, J.S., Berezin, A.A., \& Ito, T. (1997). Control of ultrafine particles from incense smoke by air cleaning electrostatic precipitators. Journal of Aerosol Science, 28.

[11] Chambre, A. (2014). Effects of carbon filtration type on filter efficiency and efficacy: Granular loose fill vs. bonded filters. Air Science.

[12] Kujundzic, E., Zander, D.A., Hernandez, M., Angenent, L.T., Henderson, D.E., \& Miller, S.L. (2005). Effects of ceiling-mounted HEPA-UV air filters on airborne bacteria concentrations in an indoor therapy pool building. Kuhn Journal of the Air \& Waste Management Association, 55, 210-218.

[13]https://commons.wikimedia.org/wiki/File:HEPA_Filter_diagram_en.svg\#media/File:HEPA_Filter_diagram_en.s $\operatorname{vg}$

[14] Liu, C., Hsu P.C., Lee, H.W., Ye, M., Zheng, G., Liu, N., Li, W., \& Cui, Y. (2015). Transparent air filter for highefficiency $\mathrm{PM}_{2.5}$ capture. Nature Communications, 6.

[15] Ren, H., Koshy, P., Chen, W., Qi, S., \& Sorrell C.C. (2017). Photocatalytic materials and technologies for air purification. Journal of Hazardous Materials, 325, 340-366.

[16] Pichat, P., Disdier, J., Hoang-Van, C., Mas, D., Goutailler, G., \& Gaysse, C. (2000). Purification/deodorization of indoor air and gaseous effluents by $\mathrm{TiO}_{2}$ photocatalysis. Catalysis Today, 63(2-4), 363-369.

[17] Beeldens, A. An environmental friendly solution for air purification and self-cleaning effect: the application of TIO2 as photocatalyst in concrete. Belgian Road Research Centre.

[18] Asadi, S., Hassan, M., Nadiri, A., \& Dyla, H. (2014). Artificial intelligence modeling to evaluate field performance of photocatalytic asphalt pavement for ambient air purification. Environmental Science and Pollution Research, 21(14), 8847-8857.

[19] Liu, X., Souzandeh, H., Zheng, Y., Xie, Y., Zhong, W., \& Wang, C. (2017). Soy protein isolate/bacterial cellulose composite membranes for high efficiency particulate air filtration. Composites Science and Technology, 138, $124-133$. [20] Ling, S., Jin, K., Kaplan, D.L., \& Buehler, M.J. (2016). Ultrathin free-standing Bombyx mori silk nanofibril membranes. Nano Letters.

[21] http://engineering.dartmouth.edu/ d30345d/courses/engs37/esps.pdf 